\title{
Buttonhole o puntura a scala di corda?
}

\author{
Lucia Barbetta, Sandra Comper, Rita Muraro \\ Emodialisi di Rovereto, Azienda Provinciale Servizi Sanitari, Trento
}

\begin{abstract}
Buttonhole technique or rope ladder technique?
Buttonhole cannulation technique (BH) was introduced by Doctor Twardowsky, a Polish nephrologist, in the seventies and was proposed in Italy and in a great part of Europe in more recent years. It consists in the placement of needles exactly in the same spot, at each treatment. An increased interest in the technique is justified by a greater attention to patients' needs and to their AVF preservation.

BH technique was introduced in the dialysis center of Rovereto in 2008. After 6 years, a two-months multicenter observational study was performed in order to compare BH cannulation and rope ladder data, concerning pain hemostasis and hematomas. With a single center retrospective analysis, infection and aneurisms were evaluated as well. $\mathrm{BH}$ group showed less pain, less infections, less hematomas and less aneurisms compared to the rope ladder group. $\mathrm{BH}$ has got some challenges regarding the need for nurses education and training, single cannulator allocation while implementing $\mathrm{BH}$ sites and strict adherence to specific clinical practice protocol in order to prevent infections. The benefits for the patients are evident and none of our patients wanted to return to previous cannulation practice.
\end{abstract}

Keywords: AVF cannulation, Buttonhole cannulation, Dialysis patient, Infection

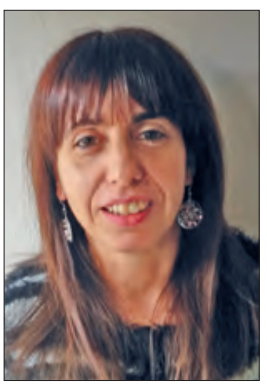

\section{Introduzione}

L'emodialisi è un indispensabile trattamento salvavita ma, al tempo stesso, appare chiaramente essere una pericolosa fonte di infezioni sia per la singolarità delle azioni invasive svolte per la sua realizzazione sia per la particolare compromissione del sistema immunitario nell'emodializzato. L'infermiere risulta essere una figura determinante nella prevenzione degli eventi avversi tramite la scrupolosa adozione delle precauzioni standard e del lavaggio delle mani e l'utilizzo dei dispositivi di protezione individuale (DPI).

Un accesso vascolare ben funzionante è un requisito indispensabile per garantire un trattamento dialitico ottimale,

Accepted: October 31, 2016

Published online: November 29, 2016

Indirizzo per la corrispondenza:

Lucia Barbetta

U.O. Emodialisi

Ospedale di Rovereto

Piazzale Santa Maria 6

38068 Trento

lucia.barbetta@apss.tn.it rappresentando tuttora il tallone d'Achille dell'emodialisi. Infatti, "l'emodialisi è associata a un alto rischio di morbidità e mortalità. Gli episodi infettivi e, in particolare, quelli correlati all'accesso vascolare contribuiscono in maniera importante a questo rischio e spiegano almeno il 15\% degli episodi di ospedalizzazione nella popolazione in trattamento sostitutivo. La fistola con vasi nativi o l'accesso dialitico protesico hanno un ridotto rischio infettivo rispetto al catetere (sia temporaneo che tunnellizzato)" (1).

Ecco il motivo per cui le Linee Guida del NKF KDOQI raccomandano I'utilizzo della fistola arterovenosa (FAV) come scelta primaria (2).

La sopravvivenza generale della FAV dipende non solo dai vasi prescelti e dalla tecnica chirurgica adottata, ma anche dalla modalità di venipuntura.

In letteratura e nella pratica clinica sono descritte 3 metodiche di venipuntura:

- puntura zonale: la puntura viene sempre eseguita nella stessa area cutanea. Può comportare la comparsa di aneurismi a carico dei vasi dello shunt e lesioni stenotiche;

- puntura a scala di corda (3): consiste nel cambiare il punto di inserzione dell'ago ad ogni seduta dialitica, utilizzando tutto il decorso cutaneo del vaso. In tal modo, la traumatizzazione e la cicatrizzazione si distribuiscono sull'intera lunghezza della vena arterializzata e si creano delle modeste dilatazioni che, tuttavia, non degenerano in aneurismi; 
- puntura buttonhole (BH) o puntura a "sito costante" $(4,5)$ : è una tecnica che prevede l'incannulamento con ago tagliente della FAV da parte dello stesso operatore per 6-8 sedute consecutive esattamente:

- nello stesso punto;

- con la stessa inclinazione;

- con la stessa profondità di penetrazione;

- con la stessa direzione.

In questo modo, si sviluppa un tratto di tunnel in tessuto cicatriziale, che consente il successivo utilizzo dell'ago smusso e, progressivamente, la graduale estensione della tecnica a tutto il personale infermieristico.

I VANTAGGI di questa tecnica possono essere così riassunti (6):

- $\quad$ riduzione degli ematomi;

- riduzione della sintomatologia dolorosa;

- $\quad$ riduzione del tempo di emostasi;

- riduzione della formazione di aneurismi nel lungo periodo;

- $\quad$ riduzione delle cicatrici;

- $\quad$ migliore estetica del braccio;

- $\quad$ eventuale addestramento all'autoincannulamento (7).

Nel nostro Centro, la puntura a occhiello è stata introdotta nel 2008, dopo un incontro con i referenti dell'Associazione EDTNA/ERCA Fil Italiana, nell'ambito della campagna di divulgazione da loro intrapresa. II crescente interesse verso questa tecnica ha, prima, stimolato una ricerca bibliografica dalla quale sono emersi dati parzialmente discordanti (8-11) e, in seguito, spinto a fare uno studio osservazionale multicentrico della durata di tre mesi.

\section{Metodo di studio}

Studio osservazionale multicentrico, con intervallo temporale da Luglio a Settembre 2014, dopo 6 anni di applicazione della metodica.

\section{Partecipanti}

Per avere un adeguato campione di pazienti trattati con la metodica $\mathrm{BH}$ sono stati coinvolti altri 4 Centri Dialisi ad Assistenza Limitata (CAL) della nostra Provincia (Tione, Arco, Borgo Valsugana, Cles), mentre, per la metodica tradizionale, è stato scelto un campione di convenienza di pazienti del Centro Dialisi di Rovereto, in quanto la tecnica con puntura a scala di corda è trasversale a tutti i Centri Dialisi del Trentino.

Sono stati presi in esame 16 pazienti in trattamento emodialitico che utilizzavano la metodica $\mathrm{BH}$ versus 16 pazienti che utilizzavano la puntura a scala di corda. I due gruppi di pazienti in studio ( $n=32$ ) erano omogenei per sesso (M. 11, F. 5), con range d'età di 34-83 anni nel gruppo della puntura

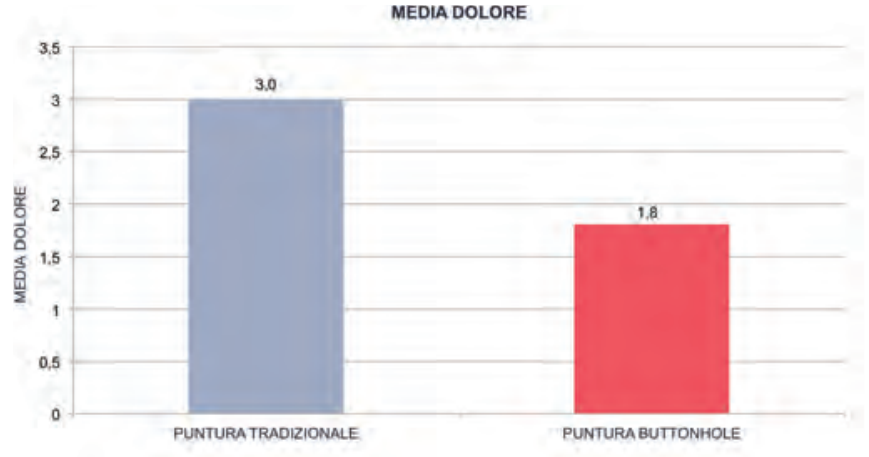

Fig. 1 - Media dolore.

tradizionale e di 35-79 anni nel gruppo $\mathrm{BH}$.

La localizzazione della FAV in entrambi i gruppi era prossimale in 6 pazienti, mediale in 4 pazienti con puntura tradizionale e in 3 nel gruppo $\mathrm{BH}$ e distale in 6 pazienti con puntura tradizionale e in 7 nel gruppo $\mathrm{BH}$.

\section{Obiettivo}

Stabilire outcome e rispettivi indicatori per comparare esiti ed eventi nelle due diverse tecniche di puntura.

\section{Outcome}

Nei tre mesi di studio sono stati raccolti dati riguardanti:

- dolore percepito alla puntura;

- tempo di emostasi;

- tempo di emostasi con utilizzo di garza emostatica;

- $\quad$ tempo di emostasi nei pazienti in trattamento con dicumarolici (con/senza utilizzo di garza emostatica);

- ematomi;

- $\quad$ infezioni rilevate con segni clinici (arrossamento, tumefazione, calore, febbre).

\section{Risultati - analisi multicentrica}

\section{Dolore alla puntura}

Utilizzando la scala VAS (Scala Visiva Analogica) da 0 a 10, dallo studio si rileva che il dolore alla puntura percepito dai pazienti trattati con la metodica $\mathrm{BH}$ è inferiore rispetto a quello percepito dai pazienti punti con la metodica tradizionale (Fig. 1).

\section{Tempo di emostasi}

Nella rilevazione del tempo di emostasi (in minuti) delle due metodiche si rileva che, nella metodica a occhiello, 10 pazienti hanno un tempo di emostasi compreso tra $3^{\prime} 18^{\prime \prime}$ e $4^{\prime} 36^{\prime \prime}, 4$ pazienti tra $5^{\prime} 00^{\prime \prime}$ e 6'48' e 1 solo paziente ha un 


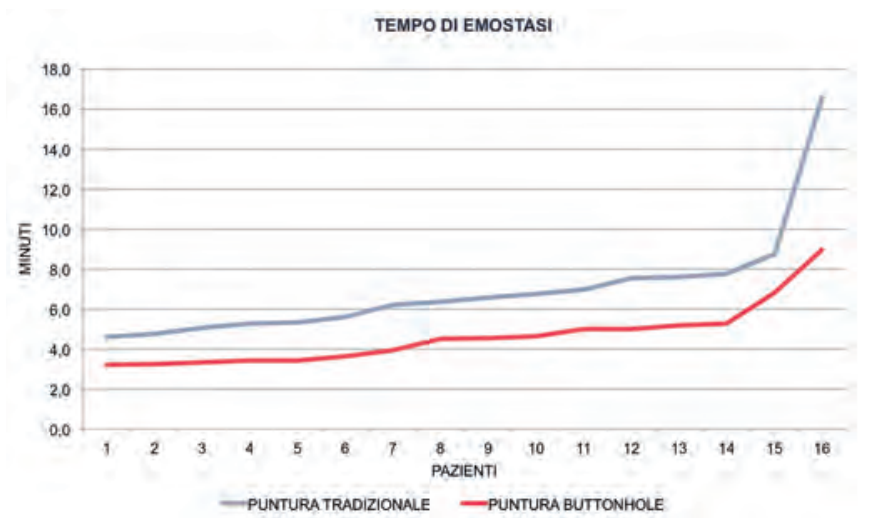

Fig. 2 - Tempo di emostasi.

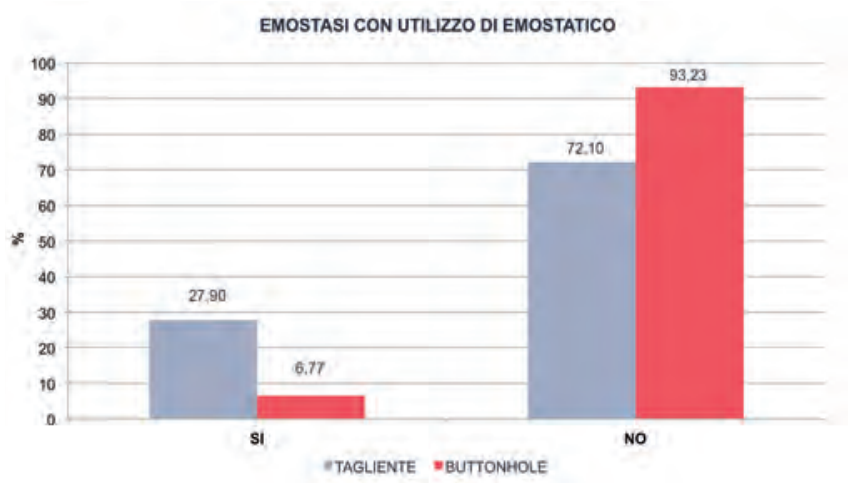

Fig. 3 - Emostasi con utilizzo di emostatico.

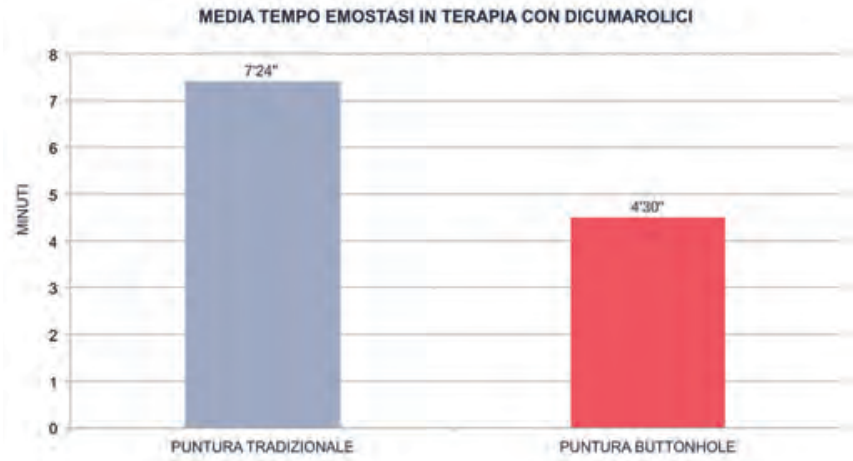

Fig. 4 - Media del tempo di emostasi nei paziente in terapia con dicumarolici (con/senza utilizzo di una garza emostatica).

tempo pari a 9'00", mentre, nella puntura "a scala di corda", nessun paziente ha un tempo di emostasi inferiore a $4^{\prime} 36^{\prime \prime}, 8$ pazienti hanno un tempo di emostasi tra $5^{\prime} 6^{\prime \prime}$ e $6^{\prime} 48^{\prime \prime}, 5$ pazienti tra $77^{\prime} 00^{\prime \prime}$ e $8^{\prime} 48^{\prime \prime}$ e uno ha addirittura un tempo di 16'36" (Fig. 2).

\section{Tempo di emostasi con utilizzo di una garza emostatica}

Prendendo in considerazione l'utilizzo di una garza emostatica, si evince che, nel $28 \%$ circa delle sedute con puntura a

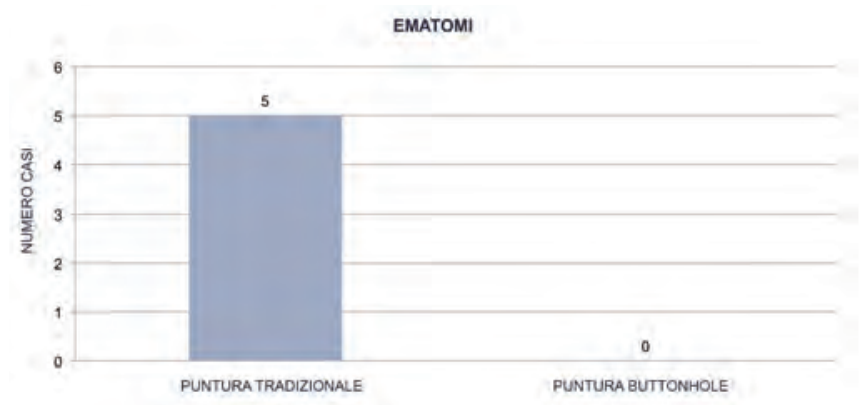

Fig. 5 - Numero di ematomi.

scala di corda, i pazienti utilizzano la garza emostatica contro il $6.7 \%$ nel gruppo che utilizza la metodica BH (Fig. 3).

\section{Tempo di emostasi in pazienti in terapia con dicumarolici}

Se si prendono in considerazione i pazienti in terapia con dicumarolici e l'eventuale utilizzo di una garza emostatica nelle due metodiche, si osserva che, nel gruppo trattato con puntura $\mathrm{BH}$, nessuno ha utilizzato la garza emostatica e si ha, comunque, una media del tempo di emostasi inferiore a quella del gruppo punto "a scala di corda", dove i pazienti hanno utilizzato la garza emostatica per il $41 \%$ delle sedute (Fig. 4).

\section{Ematomi}

Prendendo in esame i casi di ematoma che si sono verificati nel lasso di tempo considerato, sono stati rilevati 5 casi nel gruppo con puntura tradizionale e nessun caso nel gruppo BH (Fig. 5).

\section{Infezioni}

In entrambi i gruppi nel periodo preso in considerazione non si è verificata alcuna infezione.

Con la consapevolezza che uno dei limiti dello studio è stato la brevità del follow-up e che l'incidenza di infezioni nella metodica BH riscontrata in letteratura (12) è maggiore rispetto a quella della puntura a scala di corda, è stata fatta un'analisi retrospettiva delle infezioni nel nostro Centro dal 2008 al 2014, dalla quale è risultata un'incidenza in controtendenza rispetto ai dati in letteratura, pari allo $0.17 \%$ o delle sedute di emodialisi per i pazienti che seguono la metodica a occhiello versus un'incidenza dello $0.32 \%$ o per i pazienti trattati con la puntura a scala di corda (Fig. 6).

Si è trattato di infezioni locali, rilevate clinicamente per la presenza dei classici sintomi di arrossamento, dolore e gonfiore. Sono state tutte trattate con antibioticoterapia locale o sistemica.

\section{Aneurismi}

In letteratura, sembrerebbe che, con la metodica $\mathrm{BH}$, il rischio della comparsa di aneurismi sia inferiore rispetto alla 


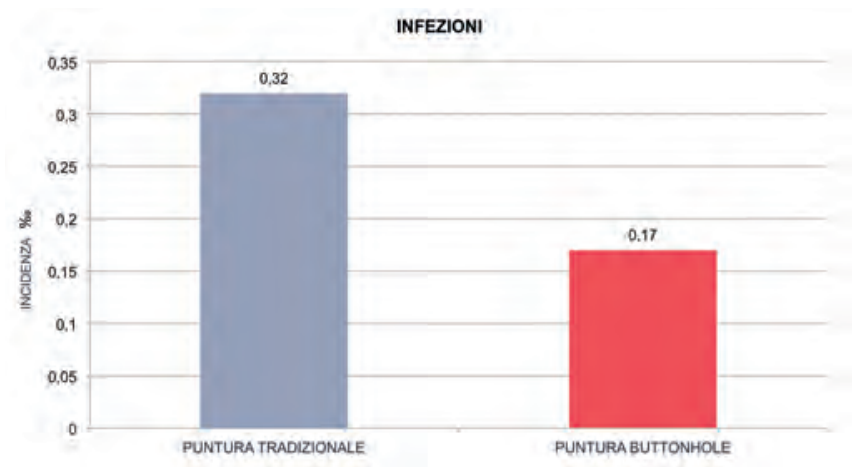

Fig. 6 - Incidenza delle infezioni.

metodica tradizionale.

È difficile, però, comparare questi studi perché spesso ciascuno di essi si basa su parametri diversi tra loro (foto, ecodoppler, misurazione della grandezza dell'aneurisma).

Anche in questo caso abbiamo preso in considerazione solo il nostro Centro Dialisi. Attraverso l'utilizzo di fotografie ripetute a distanza di tempo, si è constatato che non si sono formati aneurismi e che non sono aumentate le dimensioni di quelli già presenti.

\section{Risultati - analisi monocentrica}

Nell'esperienza quotidiana, oltre agli aspetti positivi della metodica $\mathrm{BH}$, sono state rilevate criticità come la puntura inefficace e la formazione di falsi tunnel. Per questo motivo, nell'anno 2015, sono stati raccolti ulteriori dati riguardanti I'accesso vascolare nei pazienti con puntura $\mathrm{BH}$ appartenenti solo al Centro di Rovereto, per poter analizzare in quale misura queste criticità siano rilevanti (Tab. I).

Il campione dei pazienti $(n=7)$ è risultato analogo a quello dello studio precedente, a eccezione di una paziente che è stata esclusa perché portatrice di un CVC permanente per sopravvenuto blocco della FAV.

I dati raccolti dal $1^{\circ}$ Maggio al 22 Novembre 2015 hanno preso in considerazione:

- lo stato della cute e la presenza di segni/sintomi che potessero indicare un'infezione;

- la presenza di difficoltà alla venipuntura con la necessità di procedere a un nuovo incannulamento con ago smusso o con l'utilizzo di un ago tagliente;

- la presenza di false vie.

Le sedute da analizzare erano 616: in 139 (22.56\%), i dati non sono stati raccolti, per cui solo 477 (77.44\%) sono state valutate. I risultati di questa raccolta hanno evidenziato che:

in nessun paziente si sono rilevati segni/sintomi di infezione; in 2 sedute $(0.42 \%)$, per lo stesso paziente, si è rilevata la presenza di due "fori" adiacenti;
TABELLA I - Criticità

\begin{tabular}{|c|c|c|c|c|c|c|c|c|c|}
\hline \multicolumn{10}{|c|}{ Criticità } \\
\hline & pz 1 & pz 2 & pz 3 & pz 4 & pz 5 & pz 6 & pz 7 & $\begin{array}{l}\text { Tot. } \\
\text { casi }\end{array}$ & $\%$ \\
\hline $\begin{array}{l}\text { Segni/sintomi } \\
\text { infezione }\end{array}$ & 0 & 0 & 0 & 0 & 0 & 0 & 0 & 0 & 0 \\
\hline $\begin{array}{l}\text { Cute di accesso } \\
\text { danneggiata }\end{array}$ & 0 & 0 & 0 & 0 & 0 & 0 & 1 & 1 & 0.42 \\
\hline $\begin{array}{l}\text { Difficoltà nella } \\
\text { puntura }\end{array}$ & 17 & 3 & 0 & 21 & 23 & 1 & 7 & 72 & 15.1 \\
\hline $\begin{array}{l}\text { Necessità nuova } \\
\text { incannulazione }\end{array}$ & 6 & 0 & 0 & 6 & 23 & 0 & 2 & 37 & 7.8 \\
\hline $\begin{array}{l}\text { Presenza di } \\
\text { false vie }\end{array}$ & 1 & 0 & 0 & 15 & 14 & 0 & 4 & 34 & 7.1 \\
\hline
\end{tabular}

- $\quad$ in $72(15.1 \%)$ sedute si è riscontrata difficoltà nella puntura. Solo in 37 di esse (51.3\%) è stato necessario procedere a un nuovo incannulamento.

- $\quad$ In 34 sedute (7.1\%) è stata segnalata la presenza di falsi tunnel e, nell' $85 \%$ dei casi, il dato riguarda due pazienti. Analizzando la casistica, si riscontra che la presenza di false vie è correlata alla difficoltà del posizionamento degli aghi e alla necessità di riposizionare più volte l'ago smusso. In nessun caso si è utilizzato l'ago tagliente nel tunnel;

- nel corso del periodo in esame due pazienti hanno manifestato alcuni problemi che hanno comportato l'abbandono di uno dei due tunnel: in un paziente è stata diagnosticata una stenosi a monte del tunnel, mentre, nel secondo paziente, la presenza di false vie ha reso la puntura difficoltosa, tanto da rendere impossibile il reperimento del vaso. Entrambi i pazienti hanno esplicitamente richiesto di procedere alla formazione di un nuovo tunnel appena possibile.

\section{Discussione}

In linea con quelli riportati in una parte della letteratura (13), i risultati acquisiti nel Centro Dialisi di Rovereto mostrano come la metodica $\mathrm{BH}$ sia da ritenersi superiore rispetto a quella tradizionale per quanto riguarda dolore percepito, tempi di emostasi ed ematomi.

Riguardo alle infezioni, i risultati del nostro lavoro mostrano un rischio di infezione inferiore rispetto alla puntura a scala di corda, in contrasto con quanto, invece, emerge da una certa letteratura $(9,12,14,15)$. È una criticità che spesso fa propendere a non utilizzare la metodica $\mathrm{BH}$ a favore di quella tradizionale; tuttavia, avendo investito risorse ed energie nella formazione del personale e nel rigoroso rispetto delle procedure, nella nostra esperienza, i dati relativi alla puntura a occhiello sono associati a una notevole riduzione delle infezioni della FAV, rispetto alla puntura a rotazione, dati paragonabili ad altre esperienze riportate in letteratura $(16,17)$. 
In alcuni centri di maggiori dimensioni del nostro, per controllare e monitorare le complicanze correlate agli accessi vascolari e perseguire esiti ottimali, è stata creata la figura infermieristica della Referente degli Accessi Vascolari (R.A.V.) con funzioni di supervisione e supporto per "la personalizzazione dell'approccio all'accesso vascolare, proponendo ai colleghi soluzioni operative per la gestione delle problematiche di puntura, assicurando, così, un alto livello di assistenza a tutti i pazienti" (18). Riteniamo che, anche senza una formale definizione di ruolo, anche nel nostro servizio il nostro agire possa essere paragonato a quello citato della RAV.

\section{Conclusione}

Secondo l'esperienza acquisita, la tecnica BH presenta criticità di tipo organizzativo, correlate al tempo necessario per l'allestimento del tunnel dove è impegnato un unico operatore, e criticità legate all'aumento del tempo necessario per l'attacco del paziente e alla necessità di attenersi scrupolosamente al protocollo per prevenire infezioni.

Anche la formazione di false vie è un aspetto critico da non sottovalutare e da monitorare per studiarne le cause.

La presenza di falsi tunnel può rendere la puntura difficoltosa, ma anche manovre di incannulamento inadeguate ( $p$. es., eccessivo numero di tentativi, forzatura nell'inserimento degli aghi) possono causarne la formazione.

Secondo il nostro pensiero, suffragato dalla bibliografia, questi inconvenienti si superano attraverso la competenza di chi è chiamato a praticare la tecnica e con un'adeguata organizzazione del servizio.

"La BH non è una variabile della puntura a scala di corda, ma è una tecnica del tutto diversa, al punto da richiedere non solo una riorganizzazione del lavoro e dei compiti dello staff, ma, soprattutto, un vero e proprio cambiamento culturale che dovrebbe riguardare non solo il personale addetto ma anche la Dirigenza Medico-Infermieristica e la Direzione Sanitaria... ma, soprattutto, dovrebbe volere... un numero limitato di pungitori in grado di praticare la puntura non solo con l'ago tagliente, ma anche con l'ago smusso... (Galli, Zollo e Cavatorta)" (19).

Riteniamo sia rilevante il dato che tutti i pazienti arruolati nello studio abbiano riscontrato effetti positivi, si siano dichiarati contenti e, a dimostrazione della scelta fatta, abbiano chiesto di poter proseguire nella metodica anche con la formazione di nuovi tunnel, in presenza di complicanze.

\section{Ringraziamenti}

Alle colleghe dell'EDTNA/ERCA, che hanno divulgato la metodica, Dr.ssa Marisa Pegoraro e Dr.ssa Raffaella Beltrandi, per averci sostenuto nell'introduzione della Tecnica e per la disponibilità a dirimere dubbi e problemi. Un grazie anche a tutti i colleghi che hanno collaborato alla raccolta dei dati.

Desideriamo, infine, ringraziare la Dr.ssa Renata Lazzeri, Infermiera addetta al controllo delle infezioni, e il Dr. Maurizio
Fauri, Coordinatore Infermieristico del Centro Dialisi di Rovereto, per il supporto dato alla stesura di questo articolo con suggerimenti, critiche e osservazioni.

\section{Disclosures}

Financial support: No financial support was received for this submission. Conflict of interest: The authors have no conflict of interest.

\section{Bibliografia}

1. Brunori G. L'infezione dell'accesso vascolare nell'emodializzato. G Ital Nefrol. 2012;29(Suppl. 56):S49-55.

2. KDOQI Clinical Practice Guidelines for Vascular Access. American Journal of Kidney Diseases. 2006; 48 (suppl 1): S176-S247. Disponibilesu: http://www.ajkd.org/issue/S0272-6386(06)X0213-5 (accesso il 26 Novembre 2016).

3. Günther Schönweiß. La Dialisi. In: ed. Momento Medico s.r.I., Salerno:1992; 109-110.

4. Twardowski ZJ. Constant site (Buttonhole) method of needle insertion for hemodialysis. Dialysis \& Transplantation. 2011;40:441-3. Disponibile su:http://onlinelibrary.wiley.com/ doi/10.1002/dat.20621/full (accesso il 26 Novembre 2016).

5. Twardowski ZJ. Utility of the buttonhole cannulation method for hemodialysis patients with arteriovenous fistulas. Nat Clin Pract Nephrol. 2007;3(12):648-9.

6. Pergolotti A, Rich E, Lock KT. The effect of the buttonhole method vs. the traditional method of AV fistula cannulation on hemostasis, needle stick pain, pre-needle stick anxiety, and presence of aneurysms in ambulatory patient on hemodialysis. Nephrol Nurs J. 2011;38(4):333-6.

7. Verhallen AM, Kooistra MP, Van Jaarsveld BC. Cannulating in hemodialysis: rope-ladder or buttonhole technique? Nephrol Dial Transplant. 2007;22:2601-4.

8. Atkar RK, MacRae JM. The buttonhole technique for fistula cannulation: pros and cons. Curr Opin Nephrol Hypertens. 2013;22(6):629-36.

9. MacRae JM, Ahmed SB, Atkar R, et al. A randomized trial comparing buttonhole with rope ladder needling in conventional hemodialysis patients. Clin J Am Soc Nephrol. 2012;7:1632-8.

10. Smyth W, Hartig V, Manickam V. Outcomes of buttonhole and rope-ladder cannulation techniques in a tropical renal service. J Ren Care. 2013;39(3):157-65.

11. Chan MR, Shobande O, Vats $\mathrm{H}$, et al. The effect of buttonhole cannulation vs. rope-ladder technique on hemodialysis access patency. Semin Dial. 2014;27(2):210-6.

12. Grudzinski A, Mendelssohn D, Pierratos A, et al. A systematic review of buttonhole cannulation practices and outcomes. Semin Dial. 2013;26(4):465-75.

13. Evans LM. Buttonhole cannulation for hemodialysis: a nursing review. Renal Society of Australasia Journal. 2012;8(3):146-51.

14. Pegoraro M. La puntura a occhiello o buttonhole: quali sono le cose che "fanno la differenza". Una revisione della letteratura infermieristica. Giornale di Tecniche Nefrologiche e Dialitiche. 2013;25(2):118-12514.

15. MacRae JM, Ahmed SB, Hemmelgarn BR. Buttonhole Cannulation Technique as the Cannulation Method of Choice. Am J Kidney Dis. 2014;63(1):165.

16. Labriola L, Crott R, Desmet C, et al. Infectious complications following conversion to Buttonhole cannulation of native arteriovenous fistulas: a quality improvement report. Am J Kidney Dis. 2011;57(3):442-8.

17. Ball LK. The buttonhole technique: strategies to reduce infection. Nephrol Nurs J 2010;37(5):473-8.

18. Beltrandi R. Gli infermieri in nefrologia:schede delle esperienze". Disponibilesu: http://www.nephromeet.com/web/procedure/protocollo.cfm?List=WsIdEvento,WsIdRisposta,WsReleas e\&c1=00098\&c2=12\&c3=1 (Accesso il 26 Novembre 2016).

19. Lombardi M, Quintaliani G. Quale operatore, per quale venipuntura, per quale paziente in emodialisi? Giornale di Tecniche Nefrologiche e Dialitiche. 2014;26(1):1-3. 